1 Running head: PREDICTIVE PROCESSING AND LANGUAGE DISORDER

2

3

4

5

6

7

8

9

1

\title{
Predictive processing and developmental language disorder
}

2

Samuel David Jones and Gert Westermann

Department of Psychology, Lancaster University

2777 words

20 This work was supported by Economic and Social Research Council International Centre

1 for Language and Communicative Development (LuCiD) [ES/S007113/1 and

2 ES/L008955/1]. We have no conflicts of interest to disclose.

3 Correspondence concerning this article should be addressed to Sam Jones,

4 Department of Psychology, Lancaster University, Lancaster, United Kingdom, LA1 4YF.

25 Email: sam.jones@lancaster.ac.uk. Telephone: +44 (0) 1524593698. 


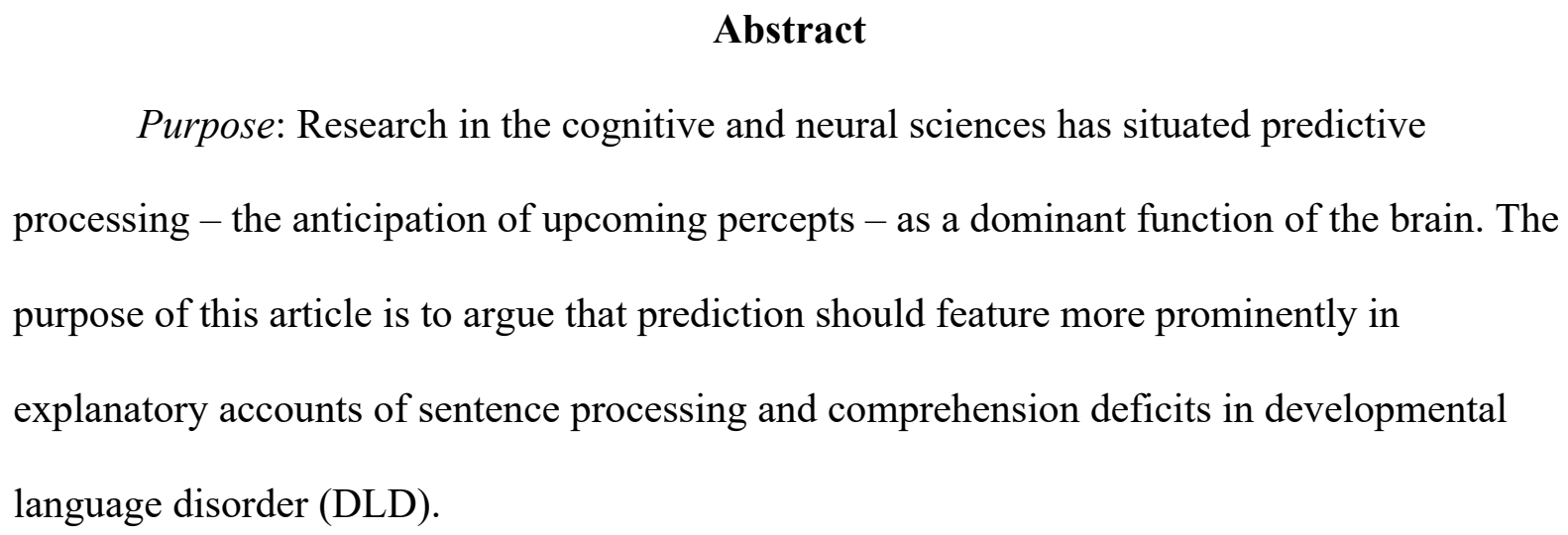

Abstract

Purpose: Research in the cognitive and neural sciences has situated predictive processing - the anticipation of upcoming percepts - as a dominant function of the brain. The purpose of this article is to argue that prediction should feature more prominently in explanatory accounts of sentence processing and comprehension deficits in developmental language disorder (DLD).

Method: We evaluate behavioural and neurophysiological data relevant to the theme of prediction in early typical and atypical language acquisition and processing. Results: Poor syntactic awareness - attributable in part to an underlying statistical learning deficit - is likely to impede syntax-based predictive processing in children with DLD, conferring deficits in spoken sentence comprehension. Furthermore, there may be a feedback cycle in which poor syntactic awareness impedes children's ability to anticipate upcoming percepts, and this in turn makes children unable to improve their syntactic awareness on the basis of prediction error signals.

Conclusion: This article offers a re-focusing of theory on sentence processing and comprehension deficits in DLD, from a difficulty in processing and integrating perceived syntactic features, to a difficulty in anticipating what is coming next. 


\section{Sentence processing and comprehension deficits in children with developmental}

\section{language disorder}

Around seven percent of English-speaking children are affected by developmental language disorder (DLD), defined as a severe language deficit in the absence of a clear biomedical cause (Bishop, Snowling, Thompson, \& Greenhalgh, 2016; Norbury et al., 2016). DLD is characterised by impairments in spoken sentence comprehension and production, although in the current article we focus on comprehension alone. This includes difficulty understanding long sentences such as the boy in the red jumper is making tea for the woman in yellow and complex sentences (e.g. passives) such as the girl was pinched by the crab (Norbury, Bishop, \& Briscoe, 2002).

Sentence comprehension deficits in DLD are commonly linked to limitations in the speed or capacity of cognitive processing (see Leonard, 2014, pp. 271-303, for review). In support of this view, children with DLD are reported to be slower than age-matched peers to make grammaticality judgements (Wulfeck \& Bates, 1995), and to identify target words during sentence listening (Stark \& Montgomery, 1995). Furthermore, performance profiles similar to those of children with DLD (e.g. the mis-processing of tense and agreement morphemes) can be elicited in typically developing children by increasing the speed of spoken sentence stimuli by 50\% (Hayiou-Thomas, Bishop, \& Plunkett, 2004).

To date, explanatory accounts of sentence comprehension deficits in DLD have placed little emphasis on predictive processing, defined as the implicit anticipation of upcoming percepts. Instead, emphasis has been on identifying the mechanisms deficient in the processing of perceived stimuli, and determining how such deficits lead to a breakdown in sentence comprehension. For instance, a recent study by Gillam, Montgomery, Evans, and Gillam (2019) used factor analysis to identify four latent variables associated with spoken sentence comprehension deficits among 117 children with DLD: (i) fluid reasoning; (ii) 
controlled attention; and (iii) long-term language knowledge; which together affect sentence comprehension by way of (iv) complex working memory. Gillam et al. (2019) provide one of the most sophisticated inquiries to date into sentence processing and comprehension deficits in DLD, due to a large sample size and a comprehensive battery of linguistic and cognitive tasks. Nevertheless, as is common of explanatory accounts in this domain, no reference is made to deficits in anticipating upcoming stimuli, with focus instead on how the constructs identified relate to processing inefficiency with respect to perceived input, and the effect that this has on sentence comprehension.

\section{Predictive processing in typically developing children}

There is, however, good reason to think that the absence of a role for prediction in explanatory accounts of sentence processing and comprehension deficits in DLD is a mistake. Research has shown the anticipation of upcoming stimuli to be an important component of typical sentence processing and comprehension. For instance, eye-tracking studies using the visual world paradigm, in which participants view an array of objects on a computer screen while listening to a sentence, show that by two to three years of age typically developing children make anticipatory eye movements towards the appropriate object (e.g. a cake) when exposed to a sentence fragment containing an informative verb (e.g. the boy eats ; Mani \& Huettig, 2012; Borovsky, Elman, \& Fernald, 2012).

Visual world paradigm data illustrate how any information available to the listener may form the basis of anticipatory language processing, from linguistic information at all levels - for instance lexical semantics (i.e. the eat/cake association) and syntax (i.e. awareness that the verb eat is in this instance transitive) - to features of the visual environment (i.e. the target and distractor images). In the current article, however, focus is on children's use of syntactic awareness to anticipate upcoming syntactic features, such as 
grammatical classes (e.g. [NOUN], [VERB]), inflectional morphemes (e.g. -s, -ing, -ed), and syntactic structures (e.g. the passive; was [PAST PARTICIPLE] by [SUBJECT]).

Electroencephalography (EEG) research has been key in isolating a neural signature associated with the apparently automatic identification of violations of syntax-based predictions made during spoken sentence exposure. The early left anterior negativity (ELAN) - a negative inflection of the recorded electrophysiological waveform at approximately 200 milliseconds after stimulus onset - is associated with the online detection of syntactic anomalies in spoken sentences such as tomorrow I will going to the park (see Friederici, 2006, for review). Evidence of ELAN components during anomalous spoken sentence exposure in children aged just two and a half suggests that the anticipation of upcoming syntactic information is a standard feature of sentence processing early in typical development, as it is in adulthood (Friederici, 2006).

The ELAN is one of three major signatures commonly discussed with respect to sentence processing, in addition to the $\mathrm{P} 600$ - a positive inflection approximately 600 milliseconds after stimulus onset associated with late sentence-level reanalysis following the detection of a syntactic anomaly - and the N400 - a negative inflection approximately 400 milliseconds after stimulus onset associated with the detection of a semantic anomaly. P600 and N400 signatures emerge earlier than the ELAN among typically developing children, suggesting that online syntax-driven anticipatory processing is a relatively advanced sentence comprehension strategy (Friederici, 2006).

\section{The benefits of syntax-based predictive processing}

Syntax-based predictive processing confers two primary advantages. First, prediction makes online sentence processing efficient by preparing the listener to rapidly resolve ambiguity and integrate perceived inputs into a comprehensible mental representation (Ferreira \& Chantavarin, 2018). Second, prediction error may drive learning, with 
unanticipated inputs eliciting heightened attention and marked increases in neural activity consistent with updates in the knowledge base guiding prediction and its underlying neural structure (Rabagliati, Gambi, \& Pickering, 2016). With respect to syntax-based predictive

123 processing, this knowledge base - the child's syntactic awareness - incorporates implicit,

124 probabilistic understanding of syntactic categories such as [NOUN] and [VERB], and of

125 distributional regularities such as progressive (i.e. is [VERB]-ing) and passive (i.e. was [PAST PARTICIPLE] by [SUBJECT]) adjacency relations. Where a perceived input does not align with predictions, updates to this knowledge base and its underlying neural structure may be made with the aim of improving the precision of future predictions (den Ouden, Kok,

$129 \&$ de Lange, 2012; Friston, 2005).

130 Our position is not, however, that syntax-based prediction is essential for either

131 sentence comprehension or the development of syntactic awareness. In any given

132 environment multiple cues (e.g. semantic and pragmatic information) determine the

133 efficiency and accuracy with which a sentence is comprehended. Furthermore, there is

134 evidence that comprehension and learning are possible in the absence of anticipation on any

135 basis (e.g. lexico-semantic or syntactic; Huettig \& Mani, 2016). In the aforementioned eye-

136 tracking work by Mani and Huettig (2012), for instance, sentence comprehension was

137 recorded even among children in lower language centiles, who made fewer anticipatory eye

138 movements towards the target in the visual array. For these reasons, our position is that

139 predictive processing has a facilitatory rather than essential role in sentence comprehension

140 and the development of syntactic awareness. We consider the implicit anticipation of

141 upcoming syntactic features to follow naturally from reaching a standard of syntactic

142 awareness, bringing with it increased sentence processing efficiency and comprehension

143 accuracy, as well as the error-driven fine-tuning of syntactic awareness.

\section{Predictive processing deficits in children with DLD}


There is reason to believe that children with DLD may fail to engage in syntax-based

146 predictive processing, and that this contributes to the sentence comprehension deficits

147 characteristic of this population. The aforementioned eye-tracking studies reporting verb-

148 information-based anticipation, for instance, show that rates of pre-emptive eye movements

149 towards the target are positively correlated with vocabulary size (Mani \& Huettig, 2012).

150 This is important because children with DLD commonly have smaller vocabularies than their 151 age-matched, language-typical peers, and so may similarly be expected to anticipate less

152 following informative verb exposure.

153 Additionally, in EEG research, ELAN components elicited in response to

154 syntactically anomalous sentences in typically developing children are often absent or

155 irregular among children with DLD, suggesting a specific difficulty in anticipating syntactic

156 information (Friederici, 2006). Importantly, EEG research often reports broadly standard

157 N400 and P600 components among children with DLD, signifying relatively minor

158 difficulties in semantic parsing and the late repair and recovery of sentence meaning. This

159 suggests that many children with DLD have not reached the standard of syntactic awareness

160 required to engage in automatic, syntax-driven anticipatory processing, and therefore

161 continue to depend on relatively immature processing strategies - i.e. semantic parsing and

162 late sentence-level reanalysis - in order to bolster sentence comprehension. While such

163 strategies may be sufficient in early development, they may not meet the linguistic challenges

164 faced by older children, namely the processing and comprehension of long or complex

165 spoken sentences. In this case, the ability to anticipate upcoming features may be a

166 significant advantage. Protracted reliance on immature processing strategies may explain

167 discrepancies in the speed of sentence processing and the accuracy of sentence

168 comprehension between many children with DLD and their age-matched, language-typical

169 peers. 
170 The basis of predictive processing deficits in DLD

Syntax-based predictive processing rests on implicit, probabilistic knowledge of

172 syntactic categories and morpho-syntactic dependencies. For most children, establishing this

173 knowledge base is straightforward, and rests on an adeptness at implicitly identifying

174 recurrent patterns in the language environment; a skill known broadly as statistical learning.

175 Typical development follows a relatively smooth trajectory from early rote-learned holophrases (e.g. daddy gone), through semi-productive slot-and-frame constructions (e.g. gone), towards abstract syntactic structures approximating the adult end state (e.g. [SUBJECT] has [PAST PARTICIPLE]) (Tomasello, 2005). In contrast, children with DLD

179 have been described as engaging in the protracted rote-learning and production of sentence

180 structures (Hsu \& Bishop, 2010). For instance, while typically developing children appear to combine prior syntactic awareness with an inference regarding a given target word's syntactic class in order to use that target accurately in novel phrases with assorted argument structures, children with DLD tend to use novel target words in new phrases that retain the argument structure of the phrase in which that target word was taught (e.g. Skipp, Windfuhr, \& Conti-Ramsden, 2002).

Similar evidence that children with DLD may fail to learn abstract distributional regularities from speech input comes from artificial grammar studies, in which learning is monitored while controlling between-class transitional probabilities, co-occurrence

189 frequencies, and the distance of dependencies (e.g. Hsu, Tomblin, \& Christiansen, 2014; see

190 Lammertink, Boersma, Wijnen, \& Rispens, 2017, for a meta-analysis of studies examining

191 statistical learning in DLD). In such studies, participants with DLD often show deficient

192 learning of abstract dependencies of the form A-X-B, where A and B are the target dependent 193 elements (e.g. [DETERMINER]-X-[NOUN]) and X is a set of intervening items of varying 194 length (e.g. the girl was pinched by [the] naughty, little [crab]). 
Relatedly, Hsu and Bishop (2014) tested the ability of seven- to eleven-year-old

children with DLD to learn linguistic and non-linguistic sequences. In the linguistic task, lists

197 of words known to the children were presented for immediate recall. Unbeknown to the

198 children, these word lists contained regularly occurring sequences which were expected to

199 elicit faster and more accurate recall if implicit sequence learning was not deficient. Poor

200 implicit learning among children with DLD was evidenced by little improvement in recall for

201 regularly occurring word sequences relative to age-matched control children. This pattern of

202 performance was, however, in line with younger children matched in grammatical ability.

203 Hsu and Bishop (2014) report correlated deficits among children with DLD in a non-

204 linguistic task measuring participants' ability to rapidly and accurately identify regular

205 changes in the location of a green creature on a computer screen. The authors argue that

206 results indicate a domain-general deficit in the acquisition of sequential information that has

207 an especially detrimental impact on the development of syntactic awareness.

\section{Summary}

The literature reviewed in this article support the following account. Some children

210 with DLD have statistical learning deficits that impact the acquisition of syntactic

211 abstractions (e.g. [NOUN], [VERB]) and morpho-syntactic dependencies (e.g. was [VERB]-

$212 e d)$. Given this deficient knowledge base, children with DLD may be unable to anticipate

213 upcoming syntactic features, such as grammatical classes (e.g. [NOUN], [VERB]),

214 inflectional morphemes (e.g. -s, -ing, -ed), and syntactic structures (e.g. the passive; was

215 [PAST PARTICIPLE] by [SUBJECT]), and may therefore be unable to rapidly resolve

216 ambiguities and integrate perceived inputs into a comprehensible mental representation.

217 Extended reliance on early-emerging sentence processing strategies - including semantic

218 parsing and the late reanalysis of sentence-level meaning - may explain deficits in the speed 
219 of sentence processing and the accuracy of sentence comprehension among children with DLD relative to age-matched, language-typical peers.

222 development of syntactic awareness. This suggests a feedback cycle in which a level of

223 syntactic awareness drives predictive processing and error coding, and error then feeds back

224 to fine-tune syntactic awareness. Error-based fine-tuning may not be a necessary precondition to the development of syntactic awareness, in the sense that without error-based fine-tuning syntactic awareness would not develop at all, but there is good evidence that it can facilitate its development (den Ouden et al., 2012; Huettig \& Mani, 2016). Indeed, the notion that expectation violation can drive learning is central to many paradigms commonly used in

229 infant and child development research, including those monitoring pupil dilation, sucking rates, gaze direction, and neurophysiological activity in response to surprising stimuli, such as objects that move in unexpected ways and unpredictable human actions (Köster, Kayhan,

232 Langeloh, \& Hoehl, 2020). Across paradigms, infants and children are more likely to attend

233 to surprising stimuli than unsurprising stimuli, plausibly in an implicit attempt to incorporate

234 unexpected behaviour into their mental models of the world. By not making syntax-based predictions, children with DLD fail by default to make erroneous predictions that generate error signals facilitating the fine-tuning of their syntactic awareness. This would be expected to further constrain the ability to make syntax-driven predictions, widening the gap in sentence processing and comprehension between many children with DLD and their agematched, language-typical peers. The relationship between syntactic awareness and the

240 ability to anticipate upcoming linguistic percepts is, therefore, likely to be reciprocal rather

241 than unidirectional.

242 Importance, clinical implications, and future research 
A deficit in the ability to anticipate upcoming syntactic features is closely linked to poor syntactic awareness, which is the hallmark of DLD. Therefore, while DLD is heterogenous, it is plausible that the current account applies to the language profiles of many affected children. This is of course not to say that syntax-based predictive processing is the only source of sentence comprehension difficulties in this population. Low vocabulary size, for instance, is just one alternative factor that may impede these children's ability to understand the sentences that they hear. Rather, the predictive processing hypothesis constitutes an important addition to the inventory of frameworks already employed to understand this complex disorder.

The account presented here suggests that improving children's ability to anticipate upcoming syntactic features will improve their spoken sentence comprehension. Despite the account outlined being theoretically novel, practically this may involve the use of existing evidence-based interventions that aim to enhance children's syntactic awareness, such as that developed by Plante et al. (2014). These authors developed a treatment programme based on the aforementioned A-X-B paradigm, and found that increasing exemplar variability, rather than input frequency, prompted a significant improvement in children's use of morphosyntax. This is likely because varying the lexical constituents within a training structure prompts children to attend to and memorise the stable syntactic elements within that structure, as well as their association (e.g. the $[\mathrm{NOUN}] \underline{\text { is }}[\mathrm{VERB}] \underline{i n g})$. The implication of the

262 account presented in the current report is that such approaches will - through improving the 263 child's implicit awareness of morpho-syntactic cooccurrence statistics - increase the child's ability to anticipate upcoming syntactic features during spoken sentence exposure, supporting rapid ambiguity resolution and the integration of perceived inputs into a comprehensible mental representation. On hearing the fragment the boy is-, for instance, the child may

267 anticipate whatever verb follows to be marked with an -ing suffix. Future experimental 
research should directly examine whether the rate of syntax-driven predictions made measured, for instance, using EEG or eye tracking methodologies - increases through highvariability programs of intervention like that developed by Plante et al. (2014).

\section{Conclusion}

Previous explanatory accounts of sentence comprehension deficits in children with

273 DLD focus on a difficulty processing and integrating perceived inputs. However, the

274 anticipation of upcoming inputs - i.e. predictive processing - has been shown to play a

275 facilitatory role in typical sentence processing and comprehension, and should, therefore,

276 feature more prominently in explanatory accounts of DLD. Suggestive evidence of predictive

277 processing deficits in children with DLD comes from EEG research, which has identified

278 irregular ELAN components in this population. Evidence of limited implicit knowledge of 279 syntactic categories and morpho-syntactic dependencies - attributable in part to statistical

280 learning problems - provides a credible basis for such deficits. Future research should test

281 whether the signals of syntax-based predictive processing - e.g. anticipatory eye movements

282 or ELAN components - strengthen or stabilise following a programme of targeted

283 intervention. 


\section{References}

285 Bishop, D. V. M., Snowling, M. J., Thompson, P. A., \& Greenhalgh, T. (2016). CATALISE:

Borovsky, A., Elman, J. L., \& Fernald, A. (2012). Knowing a lot for one's age: Vocabulary skill and not age is associated with anticipatory incremental sentence interpretation in

den Ouden, H. E. M., Kok, P., \& de Lange, F. P. (2012). How prediction errors shape

Friederici, A. D. (2006). The neural basis of language development and its impairment.

Ferreira, F., \& Chantavarin, S. (2018). Integration and prediction in language processing: A synthesis of old and new. Current Directions in Psychological Science, 27(6), 443-448. https://doi.org/10.1177/0963721418794491 Neuron, 52(6), 941-952. https://doi.org/10.1016/j.neuron.2006.12.002

Friston, K. (2005). A theory of cortical responses. Philosophical Transactions of the Royal Society B: Biological Sciences, 360(1456), 815-836. https://doi.org/10.1098/rstb.2005.1622

Gillam, R. B., Montgomery, J. W., Evans, J. L., \& Gillam, S. L. (2019). Cognitive predictors of sentence comprehension in children with and without developmental language disorder: Implications for assessment and treatment. International Journal of SpeechLanguage Pathology, 21(3), 240-251. https://doi.org/10.1080/17549507.2018.1559883

Hayiou-Thomas, M. E., Bishop, D. V. M., \& Plunkett, K. (2004). Simulating SLI: General 
cognitive processing stressors can produce a specific linguistic profile. Journal of Speech, Language, and Hearing Research, 47(6), 1347-1362. https://doi.org/10.1044/1092-4388(2004/101)

Hsu, H. J., Tomblin, J. B., \& Christiansen, M. H. (2014). Impaired statistical learning of nonadjacent dependencies in adolescents with specific language impairment. Frontiers in Psychology, 5. https://doi.org/10.3389/fpsyg.2014.00175

Hsu, Hsinjen Julie, \& Bishop, D. V. M. (2010). Grammatical difficulties in children with specific language impairment: Is learning deficient? Human Development, 53(5), 264277. https://doi.org/10.1159/000321289

Hsu, Hsinjen Julie, \& Bishop, D. V. M. (2014). Sequence-specific procedural learning deficits in children with specific language impairment. Developmental Science, 17(3). https://doi.org/10.1111/desc.12125

Huettig, F., \& Mani, N. (2016). Is prediction necessary to understand language? Probably not. Language, Cognition and Neuroscience, 31(1), 19-31. https://doi.org/10.1080/23273798.2015.1072223

Köster, M., Kayhan, E., Langeloh, M., \& Hoehl, S. (2020). Making sense of the world: Infant learning from a predictive processing perspective. Perspectives on Psychological Science, 174569161989507. https://doi.org/10.1177/1745691619895071

Lammertink, I., Boersma, P., Wijnen, F., \& Rispens, J. (2017). Statistical learning in specific language impairment: A meta-analysis. Journal of Speech, Language, and Hearing Research,60(12), 3474-3486. https://doi.org/10.1044/2017_JSLHR-L-16-0439

Leonard, L. B. (2014). Children with specific language impairment (2nd ed.). Massachusetts: 

and Performance, 38(4), 843-847. https://doi.org/10.1037/a0029284

Norbury, C. F., Bishop, D. V. M., \& Briscoe, J. (2002). Does impaired grammatical comprehension provide evidence for an innate grammar module? Applied Psycholinguistics. https://doi.org/10.1017/S0142716402002059

Norbury, C. F., Gooch, D., Wray, C., Baird, G., Charman, T., Simonoff, E., ... Pickles, A. (2016). The impact of nonverbal ability on prevalence and clinical presentation of language disorder: evidence from a population study. Journal of Child Psychology and Psychiatry, 57(11), 1247-1257. https://doi.org/10.1111/jcpp.12573

Plante, E., Ogilvie, T., Vance, R., Aguilar, J. M., Dailey, N. S., Meyers, C., ... Burton, R. (2014). Variability in the language input to children enhances learning in a treatment context. American Journal of Speech-Language Pathology, 23(4), 530-545. https://doi.org/10.1044/2014_AJSLP-13-0038

Rabagliati, H., Gambi, C., \& Pickering, M. J. (2016). Learning to predict or predicting to learn? Language, Cognition and Neuroscience, 31(1), 94-105. https://doi.org/10.1080/23273798.2015.1077979

Skipp, A., Windfuhr, K. L., \& Conti-Ramsden, G. (2002). Children's grammatical categories of verb and noun: A comparative look at children with specific language impairment (SLI) and normal language (NL). International Journal of Language \& Communication Disorders, 37(3), 253-271. https://doi.org/10.1080/13682820110119214

Stark, R., \& Montgomery, J. (1995). Sentence processing in language-impaired children under conditions of filtering and time compression. Applied Psycholinguistics, 16(2), $137-154$.

Tomasello, M. (2005). Constructing a language: A usage-based theory of language acquisition. Cambridge, Massachusetts: Harvard University Press.

Wulfeck, B., \& Bates, E. (1995). Grammatical sensitivity in children with language 
impairment (Technical Report CND-9512). San Diego. 